\title{
Approche dépendancielle de la coordination des compléments du verbe en ancien français
}

\author{
Nicolas Mazziotta \\ Université de Stuttgart - Institut für Linguistik/Romanistik \\ nicolas.mazziotta@ulg.ac.be
}

\begin{abstract}
Dans cette contribution, nous proposons une modélisation de la coordination des compléments du verbe en ancien français (désormais « afr. »). Nous adoptons ici une définition large du terme complément, qui englobe notamment le sujet, l'objet, mais également les compléments périphériques non régis. Nous avons choisi un cadre dépendanciel pour traiter le problème. Le choix de ce cadre (par opposition à une approche en constituants immédiats) est considéré ici comme une donnée qui ne sera pas discutée. La question fondamentale qui nous occupera principalement est celle de la position hiérarchique de la " conjonction de coordination » dans un modèle de ce type.

Intuitivement, on peut considérer que les constructions coordonnées en afr. ne sont pas très différentes des constructions similaires en français moderne et dans les autres langues européennes. Toutefois, certains emplois de la conjonction et en afr. ne seraient pas jugés grammaticalement corrects s'ils était transposés au français moderne. C'est le cas de la construction cel pris et celle summe d'argent dans l'ex. 1, car les deux conjoints désignent la même réalité.
\end{abstract}

(1) cel pris et celle summe d'argent doit li glise Saint-Donis paier a mun saingor Wilhame (Charte de la collégiale Saint-Denis, $1278: 1.8$ )

Ce type de binôme synonymique (ou couple synonymique) a surtout été étudié dans ses rapports avec l'activité pratique de traduction. La relation sémantique entre les synonymes est variable, mais il est généralement admis que ce type de binôme a pour effet de clarifier la traduction (Buridant, 1980). Du point de vue de la structure morphosyntaxique de la construction, Claude Buridant, suivant ici Malkiel, propose la définition minimaliste suivante : « séquence de deux synonymes appartenant en principe à la même catégorie grammaticale et placés sur le même plan de la hiérarchie syntaxique » $(1980: 5)$. Nous étudierons cette sorte de coordination et nous la comparerons à des cas qui ne montrent a priori pas de différence apparente entre les variétés modernes et anciennes. Notre exposé sera initialement centré sur la description de la coordination d'un point de vue général et nous le focaliserons progressivement sur les spécificités de l'afr.

Dans la section 1, nous passons en revue les principales modélisations pour analyser la coordination proposées dans un cadre dépendanciel. Dans la section 2, nous soulignons brièvement la particularité qu'ont nombre de constructions en afr. d'être grammaticales sans qu'aucune conjonction ou préposition ne soit employée. Dans la section 3, nous examinons la juxtaposition et l'apposition en afr. Nous montrons que ces deux constructions peuvent être précisées des points de vue syntaxique et sémantique par l'emploi de la même conjonction et - un mécanisme très proche de celui qu'Alain Lemaréchal nomme spécification (1997); elles forment ainsi deux sortes de coordination.

\section{Descriptions de la coordination dans un cadre dépendanciel}

Dans un premier temps, nous donnerons un aperçu de la manière dont la coordination a été décrite dans les principaux modèles dépendanciels. Du fait que la notion même de dépendance a une acception variable d'une théorie à l'autre, il sera nécessaire de présenter brièvement les différentes définitions de ce concept. Quelle que soit cette définition, la nature même de la modélisation nous amène à poser la question de la hiérarchie entre les unités lexicales. 
Nous synthétiserons cette section (1.4) en résumant les différentes options descriptives qu'offrent les modèles examinés. Bien que cette section prenne un point de vue général sur la question et que les choix les mieux adaptés à l'analyse de l'ancien français seront sélectionnés ultérieurement, nous utiliserons ici un exemple simple de cette langue pour présenter les différents modèles :

(2) Prenez mon escu et ma lance (Béroul : v. 3586)

\section{1 À la source : Lucien Tesnière}

Il paraît naturel de commencer notre tour d'horizon par la description qu'en fait Lucien Tesnière, qu'on peut considérer comme le fondateur de l'approche dépendancielle moderne. Nous rappelons ici les concepts les plus utiles à notre présentation.

À la base de la théorie de Lucien Tesnière se trouve le concept de connexion, qui représente la relation de dépendance unissant un terme principal à ses subordonnés. Cette relation est "verticale » et le terme principal, graphiquement positionné au-dessus de ceux qui en dépendent dans les schémas de l'auteur (ses stemmas), a une position hiérarchique plus importante. Bien qu'il apparaisse clairement dans l'exposé de Tesnière, le principe de dépendance n'a pas été défini en tant que tel par l'auteur. Néanmoins, Paul Garde (1981 : 159-160) en propose la définition suivante :

[T] oute relation syntaxique (entre morphèmes dans le mot, entre mots dans la phrase) s'établit entre deux termes dont l'un, subordonné, dépend de l'autre, principal. Le terme principal (s'il s'agit de mots, le mot principal) est celui qui contient l'information [160] sur les rapports syntaxiques entre l'ensemble constitué par le principal et son subordonné et l'environnement de cet ensemble. (Garde, 1981 : 159160)

On peut résumer cette définition en employant les termes d'Igor Mel'čuk (2009: 25-16), auquel nous reviendrons: le terme principal est celui qui fixe la distribution du syntagme, la capacité qu'a ce dernierde se trouver lui-même dans cette position de subordonné. Cette capacité est nommée la valence passive. Ainsi, dans frm. un très grand garçon, la valence passive de très grand détermine que ce syntagme peut dépendre d'un nom. Étant donné que ce potentiel est davantage lié à grand qu'à très, grand est considéré comme le terme principal.

Au cœur de sa Syntaxe structurale, Lucien Tesnière (1965 : ch. 134 sv.) élabore le concept de jonction. C'est à l'aide de cette dernière qu'il décrit les constructions coordonnées. La jonction est une relation « horizontale », qui relie deux mots hiérarchiquement équivalents (Tesnière, 1965 : ch. 135). Dans le cadre de la syntaxe des dépendants du verbe, la jonction correspond ainsi au mécanisme structurel qui permet de multiplier les actants. Un exemple simple en est donné dans le stemma 248 du livre de Tesnière (adapté à l'ex. 2 dans la fig. 1).

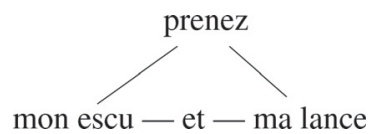

Figure 1

La représentation graphique (naturellement bidimensionnelle) étant très importante aux yeux de Tesnière, ce dernier ajoute :

Deux noeuds jonctés n'en conservent pas moins leur connexion verticale semblable. Il en résulte que l'ensemble graphique formé par leurs deux connexions verticales et le trait de jonction constitue toujours un triangle (1965: ch. 136, §4)

D'un point de vue graphique, la conjonction (le jonctif dans la terminologie de Tesnière) est placée directement sur le trait horizontal de jonction. Quand la conjonction n'est pas présente, la représentation est semblable, à ceci près que le trait de jonction n'est pas interrompu. Tesnière parle de " jonction sans jonctif» (ch. 137). 


\subsection{Approche unidimensionnelle d'Igor Mel'čuk}

Dans sa Théorie Sens-Texte (TST), Igor Mel'čuk a mis au point un ensemble de critères hiérarchisés permettant de repérer les relations de dépendance, d'en identifier le terme principal (nommé gouverneur, angl. governor) et de distinguer les relations d'ordre différent (Mel'čuk, 2009:25-40). Ainsi, en ce qui concerne l'identification du gouverneur, trois critères sont mobilisés avec une prévalence décroissante. Le critère syntaxique est le plus important et supplante les deux autres critères : la valence passive (voir 1.1 supra) du syntagme formé par le gouverneur et ce qui en dépend doit correspondre à la valence passive du gouverneur lui-même. Si ce critère se révèle insuffisant, le critère morphologique est mobilisé : de ce point de vue, c'est le gouverneur qui contrôle ou subit les phénomènes d'accord ou de rection entre le syntagme et son contexte syntaxique (par exemple, dans je veux qu'il vienne, le gouverneur de la subordonnée est vienne, car son mode est conditionné par veux). Si ces deux critères échouent à mettre en lumière le gouverneur, le critère sémantique est employé. Le gouverneur est un meilleur échantillon de la classe référentielle dénotée par le syntagme que ne le sont ses subordonnés ; par exemple, la lexie tarte Tatin désigne davantage une tarte qu'une Tatin... Enfin, il faut souligner les limites de ces critères, qui sont inopérants face à des formes comme plateau-repas ou bleu-vert.

Dans ce cadre, Igor Mel'čuk définit la coordination comme une relation de dépendance similaire aux autres. La coordination est définie à la fois d'un point de vue syntaxique et sémantique. Selon l'opinion de l'auteur, les membres coordonnés ne dépendent pas l'un de l'autre d'un point de vue sémantique (ils sont équivalents), mais le second membre dépend du premier d'un point de vue syntaxique. Igor Mel'čuk décrit la relation en ces termes (1988:41, nous traduisons) :

1. Dans un syntagme de la forme $X$ et $Y$, aucun élément ne demeure « indépendant » (c'est-à-dire non lié à un autre élément) [...]

2. Dans le syntagme $X$ et $Y$, la conjonction peut en être la tête [= le gouverneur], car la distribution du syntagme est déterminée par les conjoints et en aucun cas par la conjonction [...]

3. X est la tête du syntagme, car la distribution de $X$ et $Y$ est celle de $X$ et en aucun cas celle de et $Y$.

4. Dans le segment et $Y$, la conjonction est la tête : elle détermine la distribution de la chaîne à un degré supérieur que ne le fait $Y$.

La dépendance entre le premier et le second membre est toutefois généralement indirecte, du fait de l'utilisation d'une conjonction (voir fig. 2 ; nous simplifions la représentation des possessifs), ce qui n'était pas le cas chez Tesnière. Selon la TST, lorsqu'il n'y a pas de conjonction, la coordination est dite « directe » et elle correspond à la juxtaposition traditionnelle.

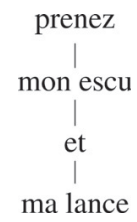

Figure 2

L'auteur lui-même admet qu'un modèle purement dépendanciel n'est pas parfaitement à-même de rendre compte de la coordination, notamment au niveau nominal. Ainsi pour reprendre son exemple, il n'y a pas de différence entre la description du syntagme anglais old men and women au sens de « de vieux hommes et de vieilles femmes » et celle de la même forme au sens de " de vieux hommes et des femmes » (Mel'čuk, 2009 : 93). Une autre limite de ce formalisme est son incapacité à modéliser les coordinations dites «elliptiques» (angl. gapping coordination; p. ex. : Jean aime Marie et Pierre, Anne) et les coordination de schémas actanciels partiels ou complets (angl. valency slot coordination; p. ex. : Jean a reçu une lettre de la part de Marie et des fleurs de la part d'Anne), qui consistent en la coordination de chaînes qui ne sont pas assimilables à des constituants (on parle de non-constituent coordination). 


\subsection{Nouveaux formalismes bidimensionnels}

Les limites du modèle de description syntaxique proposé par la TST sont liées à l'unidimensionnalité du formalisme, qui va de pair avec la projectivité. Cette dernière est posée comme une contrainte formelle majeure à laquelle la TST s'efforce de ne pas déroger. Cependant, comme Sylvain Kahane le fait remarquer, « la subordination et la coordination sont deux opérations linguistiques orthogonales et nous avons besoin d'un formalisme bidimensionnel pour rendre compte de cela [...] (Kahane, 1997 : §5.5).

\subsubsection{Arbres à bulles}

Le concept de bulle, en tant qu'objet formel représentant des groupes de nœuds pouvant être imbriqués les uns dans les autres, est présenté dans Kahane, 1997. Les éléments regroupés dans une bulle sont liés entre eux par deux types de relations : 1/ soit une relation de dépendance (le concept est défini d'un point de vue strictement formel) ; 2/ soit une relation d'imbrication. De ce fait, les bulles représentant les relations de coordination permettent de grouper des sous-bulles sans qu'aucune relation de dépendance ne soit posée entre elles. L'avantage de ce modèle est qu'il permet de traiter aisément les coordinations elliptiques et les coordinations de schémas actanciels, mais nous nous focaliserons essentiellement ici sur la position hiérarchique de la conjonction de coordination.

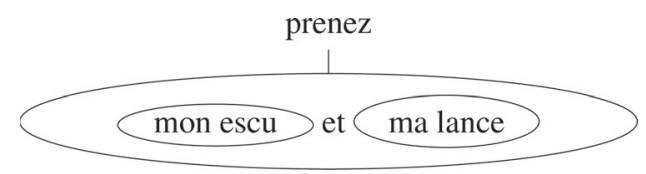

Figure 3

Observons la fig. 3. La position hiérarchique exacte de la conjonction demeure en quelque sorte floue : il s'agit simplement d'un nœud de même niveau que les membres coordonnés (notons que le lien de dépendance qui relie la bulle à son gouverneur est unique et suppose l'équivalence fonctionnelle des conjoints).

\subsubsection{Entassements paradigmatiques}

Les entassements paradigmatiques sont hérités de l'analyse en grille proposée par l'école d'Aix pour la représentation des productions orales (Blanche-Benveniste/Jeanjean, 1987 : 167-171). L'objectif principal de ce procédé, initialement pensé comme un outil pratique et non une forme de modélisation, est de rendre compte des structures interrompues et des reformulations fréquentes dans les textes oraux. Sylvain Kahane et Kim Gerdes (2009) proposent de faire usage de ce même outil pour décrire la coordination et l'apposition (une idée déjà exprimée dans Bilger, 1999, mais sans formalisation). Le modèle des entassements paradigmatiques peut être utilisé pour capturer la coordination (fig. 4) et le principe généralisé de l'analyse peut être formulé comme suit :

Nous considérons qu'un segment Y d'un énoncé s'empile avec le segment X qui le précède si Y a la même position syntaxique (i. e. : fonction) que $\mathrm{X}$ (Kahane et Gerdes, 2009 : $\$ 4$, nous traduisons)

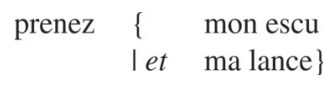

Figure 4

La forme en italique et constitue un marqueur paradigmatique. Le mot en question est considéré comme étant lié aux membres coordonnés, mais sa position syntaxique exacte n'est pas déterminée :

[...] les marqueurs paradigmatiques et ou ou, appelés d'ordinaire conjonctions de coordination, sont en relation syntagmatique exclusive avec les membres coordonnés et ne jouent aucun rôle au niveau de la combinaison des membres avec leur contexte, 
Il est clair que les concepts de bulle et d'entassement peuvent être combinés, de sorte que la formalisation tienne compte de trois types de relations: 1/ les dépendances à proprement parler, organisées hiérarchiquement dans un arbre similaire à ceux proposés par Mel'čuk ; 2/ les relations paradigmatiques, orthogonales aux premières; 3 / les relations de marquage paradigmatique (entre les marqueurs et les conjoints, voir Kahane, à paraître). Si nous combinons ces relations dans un seul schéma (fig. 5), nous voyons comment se combinent ces relations : les flèches continues représentent les dépendances, le trait double représente le lien paradigmatique et les flèches discontinues figurent les interactions qui impliquent les marqueurs paradigmatiques.

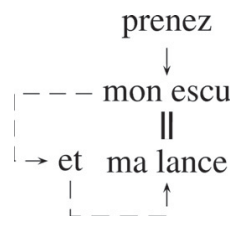

Figure 5

\subsubsection{Word grammar}

La Word grammar de Richard Hudson (2010 : 147) pose une définition sémantique de la dépendance, selon laquelle un terme subordonné précise le sens de son gouverneur.

Si l'on en croit les formulations récentes du modèle dépendanciel de la Word grammar, la conjonction de coordination est considérée comme étant dépourvue de gouverneur (elle ne dépend d'aucun autre terme). Cette conjonction est elle-même le gouverneur des deux membres coordonnés. Toutefois, la théorie ne rechigne pas à ce qu'un terme dépende de plusieurs gouverneurs simultanément. Ainsi, les compléments coordonnés sont également considérés comme dépendant du verbe (voir fig. 6).

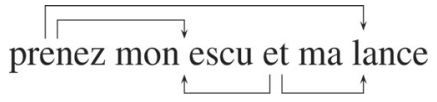

Figure 6

Dans un environnement théorique semblable, une autre option est fournie par Andrew Rosta (2006 : 189191), qui fait de la conjonction le gouverneur des compléments du verbe, dont la conjonction dépendrait directement (fig. 7).

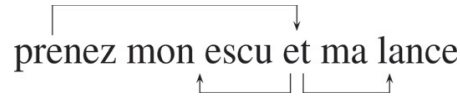

Figure 7

\subsection{Synthèse des options descriptives}

Les différents modèles que nous avons décrits présentent tous des différences qui concernent la position hiérarchique de la conjonction. Cette dernière peut en effet dépendre de différentes unités suivant les analyses, à savoir :

- la relation de coordination elle-même (Tesnière, 1965);

_ rien du tout (Hudson, 2010 ; Kahane, 1997 ; Kahane/Gerdes, 2009) ;

- le premier membre coordonné (Mel'čuk, 1988); 
- le premier membre coordonné, mais dans un jeu de relations différent de la dépendance syntaxique (Kahane, à paraître) ;

- le verbe (Rosta, 2006).

D'autre part, de la conjonction elle-même peut dépendre :

- rien du tout (Tesnière, 1965);

- $\quad$ [indéfini] (Kahane, 1997 ; Kahane/Gerdes, 2009);

- les deux membres coordonnés (Hudson, 2010 ; Rosta, 2006);

- le membre suivant (Mel'čuk, 1988);

- le membre suivant, mais dans un jeu de relations différent de la dépendance syntaxique (Kahane, à paraître).

En ce qui concerne le concept de dépendance, nous retenons la définition qu'en donne Mel'čuk, qui nous est celle qui prend en compte le plus de paramètres de manière raisonnée. En effet, comparée à la définition syntaxique de Garde (1.1) et celle, sémantique, de Hudson (1.3.3), elle intègre ces deux aspects et les hiérarchise, tout en ajoutant le critère morphologique.

Du fait que ce premier choix implique que les mots dépendent les uns des autres et qu'un mot ne peut donc dépendre d'une relation, nous devons écarter la description de Tesnière.

\section{Sous-spécification segmentale en ancien français}

Les principales descriptions syntaxiques de l'afr. (Foulet, 1968; Moignet, 1988; Ménard, 1994 ; Buridant, 2000) abordent de manière assez sommaire la question des structures coordonnées. L'essentiel de leur contribution consiste en une liste des différentes conjonctions et de leurs valeurs sémantiques et énonciatives, tout en se focalisant essentiellement sur la coordination des énoncés. La volumineuse thèse de Gérald Antoine (1958: 1962) est à cet égard beaucoup plus riche et contient de nombreux exemples classés. Toutefois, si elle reste encore utile à l'heure actuelle, force est de constater qu'elle met assez peu l'accent sur la formalisation de l'analyse de la relation.

Nous utiliserons le concept de spécification, telle que définie à la section suivante, et nous montrerons que l'afr. a de nombreuses constructions sous-spécifiées d'un point de vue segmental. Nous verrons comment les différentes options descriptives présentées dans la section 1 peuvent être évaluées au regard de cette propriété.

\subsection{Concepts de relation minimale et de spécification}

D'après les travaux d'Alain Lemaréchal, chaque relation syntaxique est sous-tendue par une relation fondamentale, dite relation minimale. Cette relation ne repose pratiquement sur aucune marque formelle. Cette approche se base sur une observation simple déjà soulignée par Lucien Tesnière (1965: ch. 152, $\S 8)$ : il arrive fréquemment que des mots contractent une relation de manière automatique, du simple fait qu'ils appartiennent à des parties du discours compatibles (l'analyse en parties du discours est partiellement une conséquence de ce fait). Par exemple, un mot comme livre et un mot comme rouge se combinent en un syntagme fonctionnant parfaitement, sans qu'il soit nécessaire d'ajouter de marque formelle (Lemaréchal, 1997 : en partic. 3 et 103). À ce stade, il suffit de remarquer qu'il existe un lien entre les formes : la question de l'orientation de la dépendance n'a pas d'importance.

Toutefois, les langues ajoutent des marques grammaticales qui permettent de distinguer aisément les fonctions : marques prosodiques, morphèmes (segmentaux), etc. De telles marques se superposent à la relation minimale et la précisent sémantiquement et syntaxiquement. Ce procédé est nommé spécification par Alain Lemaréchal (1997: 107-114). Les spécifications se combinent généralement en de complexes 
ensembles de marques de types différents (ensembles dont l'analyse ne peut se faire qu'en tenant compte du contexte). L'organisation de ces marques permet non seulement l'identification du gouverneur, mais également la différenciation des relations. Ainsi, les contraintes formelles qui portent sur les dépendants du verbe sont généralement impliquées par le choix du lexème verbal (et non le contraire).

Dans une optique variationnelle (qui inclus l'approche diachronique), les spécifications d'une relation syntaxique sont autant de variables. L'exemple (trivial) de l'évolution du sujet est révélateur : le sujet en latin est marqué par une désinence spécifique correspondant à un cas dit nominatif. En français moderne, par contre, le sujet est marqué par sa position dans la proposition. Ce résultat est l'aboutissement d'une tendance de positionnement du sujet par rapport au verbe. Dès lors que cette spécification séquentielle est devenue essentielle pour l'expression de la relation, elle est du même coup devenue obligatoire.

\subsection{Sous-spécification segmentale en ancien français}

Comme nous l'avons déjà fait remarquer dans Mazziotta, 2009 : ch. 3, on aurait toutefois peine à trouver une spécification obligatoire pour chaque fonction de l'ancienne langue. Plusieurs phénomènes très connus témoignent de cette faculté qu'a l'afr. de fonctionner sans spécification segmentale. En voici quatre exemples. Premièrement, les structures nominales exprimant le bénéficiaire sont généralement indirectes, c'est-à-dire marquées par une spécification segmentale prenant la forme d'une préposition $a$ (Mazziotta, 2009: 108). Toutefois, cette préposition peut être absente (Moignet, 1988: 296). Deuxièmement, les structures exprimant une relation « génitivale » sont le plus souvent marquées par la préposition de, mais cette spécification n'est pas obligatoire quand le possesseur est un être humain (Moignet, 1988 : 94 ; Mazziotta, 2009 : 126-127). Troisièmement, la subordination est généralement marquée à l'aide d'une conjonction, mais la parataxe est courante, y compris pour les relatives (Moignet, 1988 : 167-168, 246, 149, etc. ; voir aussi Glikman, 2009 pour une étude approfondie). Enfin, pour le cas qui nous occupe, la coordination est fréquemment « implicite», sans marqueur explicite. Par ailleurs, même dans le cas où les prépositions et les conjonctions sont employées, elles peuvent avoir des valeurs syntaxiques et sémantiques très différentes d'un emploi à l'autre (Moignet, 1988: 192). Les spécifications sont donc floues.

Les quelques exemples donnés ci-dessus impliquent que la spécification segmentale est loin de suffire à assurer le fonctionnement syntaxique de l'afr., qui repose au moins autant sur les catégories sémantiques et les facteurs situationnels et contextuels que sur ces marques segmentales.

\subsection{Conséquences}

En conséquence, le modèle dépendanciel choisi pour décrire l'afr. doit permettre le traitement des morphèmes de spécification segmentale sans nécessairement en faire des gouverneurs, comme c'est le cas dans la plupart des approches modernes. Les conjonctions et les prépositions sont, dans un nombre de cas trop fréquents pour être anecdotiques, optionnelles. Il est donc inconcevable de considérer a priori que les mots grammaticaux sont les gouverneurs de syntagmes prépositionnels ou conjonctionnels, car ils ne déterminent pas complètement la « valence passive » de la structure où ils se trouvent, ce qui constitue le critère le plus important pour déterminer la direction d'une dépendance (Mel'čuk, 2009 : 27-28). Il est toutefois clair que de nombreuses unités grammaticales sont effectivement obligatoires (Moignet, 1988 : 293), mais cela n'est en rien automatique et la formalisation de la description de l'afr. est trop peu avancée pour qu'on puisse se prononcer fermement dans tous les cas de figure, d'autant que la contrainte impliquant la présence obligatoire de certains dépendants est une des plus difficile à évaluer. Il paraît plus raisonnable de maintenir la description au niveau de la relation minimale tant que la direction dépendance ne peut être évaluée.

En conséquence, si nous voulons étudier la position de la conjonction de coordination, il est essentiel que le modèle choisi laisse cette position indéfinie. Il semble dès lors que les modèles des arbres à bulles et des entassements paradigmatiques soient les plus adéquats. 


\section{La coordination : une juxtaposition ou une apposition spécifiée}

Dans cette section, nous montrons qu'il existe deux sortes de coordinations en afr. : la première peut être considérée comme un cas spécial de juxtaposition (section 3.1). En nous appuyant sur l'équivalence structurale de la juxtaposition et de l'apposition, nous montrons dans la section 3.2 qu'il existe un second type de coordination, qui peut être envisagée comme un cas spécial d'apposition.

\subsection{Juxtaposition spécifiée}

Étant donné que la coordination peut être non spécifiée, nous suivons la piste ouverte par Gérald Antoine $(1958,461)$ en nous focalisant dans un premier temps sur ce qu'on pourrait nommer « coordination implicite », c'est-à-dire les cas où il n'y a pas de conjonction. Cette approche nous permettra de ne pas lui accorder trop d'importance d'emblée.

\subsubsection{Types d'arguments}

Jusqu'à présent, nous avons utilisé les termes coordination et juxtaposition de manière assez intuitive et traditionnelle. Employons-nous à les définir de manière plus précise, en commençant par le terme juxtaposition. Nous nous focalisons uniquement sur ce phénomène au sein de la proposition (et non entre les propositions). La question que nous posons pour orienter notre définition est la suivante : quels critères fondent la différence entre la juxtaposition et l'emploi simultané de compléments d'un même verbe dans un cadre dépendanciel ?

Du point de vue de la sémantique de la syntaxe, le verbe, envisagé de manière générale, est une unité lexicale dont la sélection par le sujet parlant contraint le rôle sémantique et l'expression morphosyntaxique de ses compléments (Lemaréchal, 1989: 102). Par exemple, dans un emploi transitif prototypique, le verbe afr. mangier aura un premier argument ayant la forme grammaticale d'un sujet (exprimé ou limité à la désinence, s'accordant normalement en personne avec le verbe, commutant avec $i l$, etc.) et exprimant l'agent. Le second argument de ce verbe aura la forme d'un objet et exprimera le patient. On peut ainsi dire que le verbe mangier régit deux types de compléments différents, chacun de ces types alignant des contraintes formelles avec un rôle sémantique (cet alignement est nommé orientation par Alain Lemaréchal). Les circonstants ne sont quant à eux pas sujets à ces contraintes de sens, de forme ou de présence. On peut ainsi formuler dans un cadre dépendanciel une "règle » universelle proche du critère- $\theta$ de la grammaire générative (Laenzlinger, $2003: 46)$ : pour chaque verbe une seule instance de chaque type d'argument peut apparaitre dans la proposition qu'il fonde. Toutefois, nous allons voir qu'il est peut-être un peu hâtif de parler de règle.

\subsubsection{Compléments juxtaposés}

Suivons le fil qui se déroule en définissant la juxtaposition comme la relation qui se manifeste, quelle que soit la langue, par la multiplication du nombre d'instance d'arguments d'un type en particulier ou l'emploi simultané de plusieurs circonstants. L'emploi simultané d'instances de types distincts ne peut être nommé juxtaposition (ex. 3 et 4).

(3) Homes, bestes, sont en repos (Eneas, v. 2163, apud Antoine, 1958 : 561)

(4) Bien li siet cele ventaille, li hiaumes, li escus, la lance (Le tournoi de Chauvency : 1. 2202)

La juxtaposition ne doit pas être envisagée comme un mécanisme permettant de transgresser une règle, mais plutôt comme un potentiel qui peut être observé. Étant donné que la multiplication des instances d'un type d'argument peut avoir lieu, il nous parait abusif de poser une règle qui stipulerait le contraire. En revanche, nous pouvons poser que lorsqu'on observe cette multiplication, on a affaire à une juxtaposition, relation qui peut s'établir sans mobiliser d'outil grammatical spécifique. 


\subsubsection{Spécification}

Selon notre approche, la conjonction de coordination qui peut être employée entre deux éléments juxtaposés est en réalité ajoutée à une relation qui pourrait exister sans elle. Nous appelons donc coordination ce type de spécification d'une relation de juxtaposition par un morphème segmental. Ainsi, l'ex. 2 peut être considéré comme une forme spécifiée de prenez mon escu, ma lance.

En conséquence, il est absurde de parler de coordination s'il n'y a pas juxtaposition (et donc si aucun type de complément n'est multiplié). De ce fait, bien que le mot et soit observable dans l'ex. 5, il n'y a pas de coordination :

(5) Nos oïemes che ke li veritauele dissent et par serement (Charte du couvent du ValBenoît, $1260: 1.10$ )

En effet : le circonstant et par serement n'est pas coordonné, car il n'y a aucun autre circonstant dans la proposition auquel il pourrait être juxtaposé. Nous considérons donc que ce et est une marque d'une spécification qui joue à un autre niveau : celui de l'articulation entre le circonstant et son gouverneur, le verbe dissent (nous ne nous étendrons pas ici au sujet de la position hiérarchique du mot par). D'un point de vue sémantique, on peut dire que l'incidence du mot et se fait vers le circonstant, auquel il confère une valeur d'insistance.

Hiérarchiquement, si la conjonction est une spécification facultative qui se combine à une relation qui n'a pas besoin d'elle pour exister, non seulement la conjonction ne peut être le gouverneur de la relation qui l'unit à l'un des conjoints (approche de Mel'čuk, cf. 1.2, supra), mais elle ne peut pas non plus se situer sur le même pied (voir 1.3.1, supra). Sauf à vouloir poser une réalisation « zéro » de la conjonction quand celle-ci ne peut être observée (option que nous souhaitons éviter), il devient évident que la conjonction est secondaire. Par ailleurs, si la conjonction était liée par une relation de dépendance aux deux conjoints, elle se trouverait nécessairement en position de gouverneur dans l'un des deux cas (en l'occurrence, celui du second conjoint, cf. Mel'čuk, 2009 : 26-27). Il devient donc clair que, si l'on veut représenter le statut secondaire de cette conjonction dans le modèle, celle-ci doit être décrite comme un dépendant du conjoint qui la suit. En d'autres termes, nous rejetons fermement la représentation classique, qui fait de la juxtaposition une forme de coordination dont la conjonction aurait été effacée (Tesnière, 1965 : ch. 137, $\S 1)$.

L'ex. 2 peut ainsi être représenté comme nous le montrons sur la fig. 8. On y observe une duplication du complément objet-patient sans que la valence du verbe n'en soit modifiée.

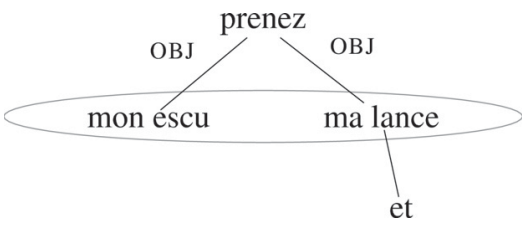

Figure 8

Dans une approche voisine des arbres à bulles de Sylvain Kahane (voir 1.3.1, supra), la relation de juxtaposition prend la forme d'une bulle. Nous ajoutons que la conjonction dépend du second conjoint. Notons qu'il ne s'agit pas exactement d'un arbre à bulles tel que Sylvain Kahane les décrit formellement, car les relations de dépendance ne peuvent normalement pas traverser les limites des bulles. Nous conservons toutefois l'idée d'une relation orthogonale aux dépendances.

On observe de nombreux exemples de la forme spécifiée de juxtaposition en cas de multiplication de compléments. À notre avis, il s'agit plus d'une question de fréquence que d'une question d'organisation grammaticale : oui, la spécification est plus fréquente, mais on ne peut en déduire qu'elle correspond à la forme de base de la relation d'un point de vue structural. La question de l'augmentation progressive de la présence de la spécification est un problème historique qu'il serait intéressant d'étudier en distinguant les circonstants (apparemment moins soumis à cette tendance) des actants. 


\subsection{Apposition spécifiée}

Nous avançons l'idée que la coordination peut en outre être envisagée comme une forme spécifiée d'apposition. Ce phénomène est propre à l'afr. et le distingue notamment du français moderne - cela ne fait aucun doute pour la variété écrite standard ; voir par ailleurs Bilger, 1999 : 263-264 pour le résultats de dépouillements de corpus de français parlé : elle ne donne aucun exemple d'apposition spécifiée.

\subsubsection{Apposition et juxtaposition}

On décrit souvent l'apposition comme un phénomène syntaxique mettant en présence deux expressions simultanées d'un même objet.

(6) Li enemy, li adversaire dunc se desrengent (Vie, martyre et miracles de saint Edmund le Roi d'Est-Anglie : 1. 2065)

(7) Tu vouloies ceste angoisse, ceste dolor, ceste painne pour nostre amor (Passion des jongleurs : 1.497$)$

(8) Adont m'arés vous retenu a vostre ami, a vostre dru (Art d'amors : 1. 1972)

Rapprochant l'apposition de la jonction (voir 1.1, supra), Lucien Tesnière a l'intuition suivante :

Le trait de jonction est de forme identique au trait d'apposition (v. chap. 69, § 7), puisque tous deux sont horizontaux. (Tesnière, 1965 : ch. 136, § 5)

Toutefois, l'approche traditionnelle fait de l'apposition une fonction, celle d'un second terme qui serait ainsi « apposé » à un autre dont il dépendrait (Grevisse et Goosse, $2007:$ § 340). Cette conception fait dire à Lucien Tesnière que bien que l'apposition soit représentée par une ligne horizontale similaire à la jonction, elle reste dépendante du mot auquel elle est apposée (1965: ch. 69, §§5-6 et ch. 139, § 6). Il est clair que c'est l'idée que l'apposition ne doit pas être envisagée au niveau de la proposition, mais au niveau inférieur du syntagme, qui sous-tend cette analyse : un terme apposé n'entretient pas de relation directe avec le verbe, mais avec un de ses compléments. Nous ne contestons pas cette analyse, mais, sauf à adopter une règle purement séquentielle, il est particulièrement difficile de déterminer quel terme est apposé et quel terme est central (gouverneur) si l'on considère qu'il y a asymétrie. En effet, d'un point de vue formel, les termes apposés peuvent partager des spécifications formelles semblables qui sont liées à leur fonction par rapport au verbe (p. ex. : l'utilisation de la préposition $a$ introduisant l'attribut de l'objet dans l'exemple 8). D'un point de vue lexico-sémantique, les termes apposés sont souvent des synonymes (ou des expressions synonymiques), comme on le voit dans les ex. 6 (enemy/adversaire) et 8 (ami/dru), ou réfèrent indubitablement au même objet, comme en témoigne l'ex. 7 (angoisse/dolor/paine).

S'il est très difficile d'établir une hiérarchie entre les termes, peut-être est-ce dû au fait qu'il n'y a pas vraiment de précédence autre que séquentielle entre eux. Or, il a déjà été avancé à diverses reprises que la différence entre l'apposition et la juxtaposition n'est pas d'ordre syntaxique : les deux constructions ne diffèrent que d'un point de vue sémantique ; par exemple, Claire Blanche-Benveniste et Sandrine Caddéo (2000) le montrent pour le français moderne dans sa variété orale, Christian Touratier (2005: 290) soutient cette analyse dans une approche « en constituants » et Kim Gerdes et Sylvain Kahane unifient les relations dans leur analyse en entassements (voir 1.3.3, supra). On peut dire simplement que ce qui sépare les deux relations est leur rapport à la coréférence : là où l'apposition implique une relation de désignation liant les deux termes au même objet ou à la même classe référentiels, la relation de juxtaposition n'implique en rien pareille similitude. Nous partageons ce point de vue et nous considérons donc que l'apposition doit être modélisée exactement de la même manière que la juxtaposition sur le plan strictement syntaxique. 


\subsubsection{Spécification}

En afr., la relation d'apposition peut être spécifiée exactement de la même manière que la relation de juxtaposition : à l'aide d'une conjonction de coordination. Cela est très clair, à la fois dans l'ex. 1 et dans l'extrait suivant (qui présente une apposition détachée entre poor et esfroi) :

(9) Poor en ont tuit et esfroi (Béroul : v. 1722)

Schématiquement, l'ex. 1 sera donc représenté par la fig. 9, dont on perçoit immédiatement le parallèle avec la fig. 8. La seule différence marquée dans le schéma prend la forme d'un trait discontinu, indiquant la relation de coréférence unissant les termes apposés tout en restant distincte des relations syntaxiques, du fait de sa nature proprement sémantique.

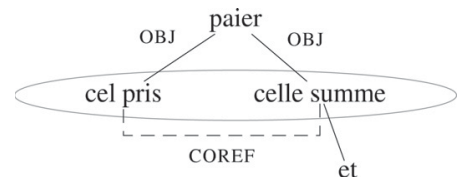

Figure 9

D'un point de vue historique, il est clair que la spécification de l'apposition a progressivement disparu du français au niveau des compléments du verbe. Ainsi, alors que l'ex. 10, qui présente une apposition au niveau de la formation du syntagme nominal, est grammatical, l'ex. 11 n'a pas le même sens et un locuteur moderne l'interprétera instinctivement en considérant que mon collègue et mon ami sont deux individus différents, à moins qu'un contexte très particulier ne permette de le réinterpréter.

(10) Je vois mon collègue et ami.

(11) Je vois mon collègue et mon ami.

Par contre, cette spécification s'est généralisée (sans toutefois devenir obligatoire) pour les juxtapositions de compléments régis. Le détail de cette progressive séparation des deux constructions reste à étudier.

\section{Conclusion}

La coordination des compléments du verbe en afr. est une forme de juxtaposition ou d'apposition spécifiée à l'aide d'une conjonction de coordination. Cette capacité commune unissant juxtaposition et apposition est spécifique à l'afr. et distingue ce dernier du français moderne.

D'un point de vue structural, la conséquence de cette spécification facultative est que la conjonction doit être analysée comme un dépendant du terme coordonné à sa suite. Bien entendu, cette analyse ne vaut que pour les langues où les relations que nous avons étudiées ne sont pas obligatoirement spécifiées par une marque segmentale.

\section{Références}

\section{Sources primaires}

Art d'amors = DEAF JacAmArtK. In NCA.

Béroul $=$ Defourques, L. M. et Muret, E. (éds). $\left({ }^{4} 1947\right)$. Béroul. Le roman de Tristan. Poème du XII ${ }^{e}$ siècle, Paris : Champion.

Charte du couvent du Val-Benoît (1260, 9 mai). Archives de l'État à Liège (Belgium).

Charte de la collégiale Saint-Denis (1278, $1^{\mathrm{er}}$ août). Archives de l'État à Liège (Belgium).

Le tournoi de Chauvency $=$ DEAF BretTournD. In NCA. 
NCA = Stein, A., Kunstmann, P. et Gleßgen, M.-D., (éds). ( $\left.{ }^{2} 2008\right)$. Nouveau Corpus d'Amsterdam. Corpus informatique de textes littéraires d'ancien français (ca 1150-1350), établi par Anthonij Dees (Amsterdam 1987), Stuttgart : Institut für Linguistik/Romanistik [textes cités identifiés à l'aide des identifiants du DEAF].

Passion des jongleurs = DEAF PassJonglGP. In NCA.

Vie, martyre et miracles de saint Edmund le Roi d'Est-Anglie = DEAF EdmK. In NCA.

\section{Études}

Antoine, G. (1958). La coordination en français. Tome I. Paris : D’Artrey.

Antoine, G. (1962). La coordination en français. Tome II. Paris : D’Artrey.

Bilger, M. (1999). Coordination: analyses syntaxiques et annotations. Recherches sur le français parlé, 15, 255-272.

Blanche-Benveniste, C. et Caddéo, S. (2000). Préliminaires à une étude de l'apposition dans la langue parlée. Langue française, 125, 60-70.

Blanche-Benveniste, C. et Jeanjean, C. (1987). Le français parlé. Transcription et édition, Paris : Didier érudition.

Buridant, C. (1977). Problèmes méthodologiques dans l'étude des traductions du latin au français au XII ${ }^{\mathrm{e}}$ siècle: le domaine lexical. Les couples de synonymes dans l'histoire de France en français de Charlemagne à PhilippeAuguste. In Buschinger, D. (éd.). Linguistique et philologie: Application aux textes médiévaux. Actes du colloque des 29 et 30 avril 1977, Paris : Champion, 293-324.

Buridant, C. (1980). Les binômes synonymiques. Esquisse d'une histoire des couples de synonymes du moyen âge au XVII ${ }^{\mathrm{e}}$ siècle. Bulletin du centre d'analyse du discours, 4, 5-79.

Buridant, C. (2000). Grammaire nouvelle de l'ancien français, Paris : Sedes.

Foulet, L. $\left({ }^{3} 1968\right)$. Petite syntaxe de l'ancien français, Paris : Champion.

Garde, P. (1981). Des parties du discours, notamment en russe. Bulletin de la Société de Linguistique de Paris, 76(1), $155-189$.

Glikman, J. (2009). Parataxe et Subordination en Ancien Français. Système syntaxique, variantes et variation. Université Paris Ouest Nanterre La Défense et Universität Potsdam. Thèse de doctorat.

Hudson, R. (2010). An introduction to word grammar, Cambridge : Cambridge University Press.

Kahane, S. et Gerdes, K. (2009). Speaking in piles. Paradigmatic annotation of a French spoken corpus. In Mahlberg, M., González-Díaz, V. et Smith, C. (éds) Proceedings of Corpus Linguistics Conference 2009, Liverpool : University of Liverpool, 16 pp. [non paginé].

Kahane, S. (1997). Bubble trees and syntactic representations. In Becker T. et Krieger H.-U. (éds). Proceedings of the 5th meeting of Mathematics of Language (MOL 5), Saarbrücken : DFKI, 70-76.

Kahane, S. (à paraître). De l'analyse en grille à la modélisation des entassements. In Caddéo S., Roubaud, M.-N., Rouquier, M. et Sabio, F. (éds). Hommage à Claire Blanche-Benveniste, Aix-en-Provence : Publications de l’Université de Provence.

Lemaréchal, A. (1989). Les parties du discours. Sémantique et syntaxe, Paris : Presses Universitaires de France.

Lemaréchal, A. (1997). Zéro(s), Paris : Presses universitaires de France.

Mazziotta, N. (2009). Ponctuation et syntaxe dans la langue française médiévale. Étude d'un corpus de chartes écrites en français à Liège entre 1236 et 1291, Tübingen : Niemeyer.

Mel'čuk, I. (1988). Dependency syntax: theory and practice. Albany : State University of New York, Albany.

Mel'čuk, I. (2009). Dependency in natural language. In Polguère A. et Mel'čuk, I. (éds), Dependency in linguistic description, Amsterdam et Philadelphia : John Benjamins, 1-110.

Moignet, G. ( ${ }^{1988)}$. Grammaire de l'ancien français. Morphologie - Syntaxe, Paris : Klincksieck

Ménard, P. ( $\left.{ }^{4} 1994\right)$. Syntaxe de l'ancien français. Bordeaux : Bière. 
(C) aux auteurs, publié par EDP Sciences, 2012

Rosta, A. (2006). Structural and distributional heads. In Sugayama, K. et Hudson, R. (éds). Word grammar. New perspectives on a theory of language structure, London et New York: Continuum, 171-203.

Tesnière, L. ( $\left.{ }^{2} 1965\right)$. Éléments de syntaxe structurale, Paris : Klincksieck.

Touratier, C. (2005). Analyse et théorie syntaxiques, Aix-en-Provence : Publications de l'Université de Provence. 